\begin{tabular}{|l|l|}
\hline FRI0156 & WORK PARTICIPATION IN PATIENTS WITH \\
SYSTEMATIC LUPUS ERYTHEMATOSUS: A \\
SYSTEMATIC REVIEW
\end{tabular}

B. Blomjous ${ }^{1,2}$, G. Gajadin ${ }^{1}$, I. Bultink ${ }^{2}$, A. Voskuyl ${ }^{2}$, L. Falzon ${ }^{3}$, J. Hoving ${ }^{4}$, M. Ter Wee ${ }^{1,2} .{ }^{1}$ Amsterdam UMC, Vrije Universiteit Amsterdam, Department of Epidemiology and Biostatistics, Amsterdam Public Health, Amsterdam, Netherlands; ${ }^{2}$ Amsterdam UMC, Vrije Universiteit Amsterdam, Department of Rheumatology and Clinical Immunology, Amsterdam Infection \& Immunity, Amsterdam, Netherlands; ${ }^{3}$ Northwell Health, Center for Personalized Health, Feinstein Institute for Medical Research, New York, United States of America; ${ }^{4}$ Amsterdam UMC, University of Amsterdam, Coronel Institute of Occupational Health, Amsterdam Public Health Research Institute, Amsterdam, Netherlands

Background: The treatment of systemic lupus erythematosus (SLE) has improved over the last decades, however, absenteeism and work disability numbers remain higher than those observed in the general population (1). SLE has its onset between the ages of 20 and 40 years, and has a major burden on the lives of patients, both mentally and financially. A recent online survey amongst 2070 European SLE patients revealed that $69.5 \%$ of patients had their careers affected due to SLE (2).

Objectives: To determine the magnitude of absenteeism and work disability in patients with SLE and to investigate the factors that might affect work participation in these patients in order to develop interventions to reduce the impact of the burden in the future.

Methods: A systematic literature search was performed to identify published articles reporting on the prevalence of work related burden, as well as the relation of having SLE on employment status, sick leave and/or presenteeism. Full-text original articles (all languages) published before April 2019 were identified by literature search performed in MEDLINE, Cochrane, Embase and CINAHL.

Results: In total, 2057 non-duplicate citations were screened after database searching and snowballing. Finally, 81 articles were included; most studies $(n=59)$ had a cross-sectional design and the remaining 22 studies had a longitudinal design. Only 15 longitudinal cohort studies reported on associations, 6 studies described a longitudinal prediction model, 2 studies reported on associations and described a prediction model, and 3 studies had a longitudinal design but only reported on prevalences over time. In total, 3500 working patients were included in the studies reporting on associations or describing a longitudinal prediction model. In the association studies, the most frequently used outcome measures were respectively being employed/probability of being employed and work loss/job cessation. Other studies used loss of days from (non)workforce activities/sick leave days, work disability, productivity loss or work entry. Most associations with work outcome were found for demographic variables and two or three disease variables, like Systemic Lupus Activity Questionnaire (SLAQ) score and depressive symptoms. This was also the case in the prediction studies. Most studies did not assess work related variables as possible predictors for work outcome.

Conclusion: A large heterogeneity was found in type of study design and outcome measures, which limits comparison with other studies. More longitudinal studies are needed to truly assess the impact of SLE on work participation, and to identify factors that could be influenced during interventions, in order to encourage work participation of these patients.

References:

[1] Bultink IE, Turkstra F, Dijkmans BA, Voskuyl AE. High prevalence of unemployment in patients with systemic lupus erythematosus: association with organ damage and health-related quality of life. J Rheumatol. 2008;35(6):1053-7.

[2] Gordon C, Isenberg D, Lerstrom K, Norton Y, Nikai E, Pushparajah DS, et al. The substantial burden of systemic lupus erythematosus on the productivity and careers of patients: a European patient-driven online survey. Rheumatology (Oxford). 2013;52(12):2292-301.

Disclosure of Interests: None declared

DOI: 10.1136/annrheumdis-2020-eular.3555

\section{FRI0157 \\ EPSTEIN BARR VIRUS BLOOD REPLICATION INCREASES DURING ACTIVE SYSTEMIC LUPUS ERYTHEMATOSUS}

P. Breillat ${ }^{1}$, A. Mathian ${ }^{1}$, S. Burrel ${ }^{2}$, M. Hié ${ }^{1}$, J. Fadlallah ${ }^{1}$, M. Pineton de Chambrun $^{1}$, F. Cohen', D. Boutolleau², F. Rozenberg ${ }^{3}$, V. Calvez ${ }^{2}$, Z. Amoura'1. ${ }^{1}$ Sorbonne Université, Assistance Publique-Hôpitaux de Paris, Groupement Hospitalier Pitié-Salpêtrière, French National Referral Center for Systemic Lupus Erythematosus, Antiphospholipid Antibody Syndrome and Other Autoimmune Disorders, Service de Médecine Interne 2, Paris, France; ${ }^{2}$ Sorbonne Université, INSERM, Institut Pierre Louis d'Epidémiologie et de Santé Publique (iPLESP), AP-HP, Hôpital Pitié-Salpêtrière, National Reference Center for Herpesviruses, Virology Department, Paris, France; ${ }^{3}$ Sorbonne Paris Cité, Assistance Publique-Hôpitaux de Paris, Hôpital Cochin, Virology Department, Paris, France
Background: A role for Epstein Barr Virus (EBV) infection in Systemic Lupus Erythematosus (SLE) pathogenesis is highly suspected. The frequency of EBV seroprevalence and DNA detection in peripheral blood mononuclear cells are increased in SLE patients compared to healthy controls.

Objectives: To analyse the relationship between EBV blood replication and SLE disease activity.

Methods: Monocentric, observational and retrospective study of SLE patients (ACR or SLICC criteria) who have had a blood EBV DNA assessment using Polymerase Chain Reaction (artus $\AA$ EBV Virus QS-RGQ assay) between 2012 and 2018. Exclusion criteria were: organ or bone marrow transplant, absence of EBV seroconversion and insufficient data. SLE clinical features, the Safety of Estrogens in Lupus Erythematosus National Assessment-Systemic Lupus Erythematosus Disease Activity Index (SELENA-SLEDAI) flare index (SFI) and therapeutic regimen on the day of EBV DNA load assessment were recorded. A SELENA-SLEDAI score $>4$ defined an active SLE. A blood EBV DNA load $\geq 125$ $\mathrm{IU} / \mathrm{mL}$ was defined as elevated.

Results: A total of 105 patients (98 women and 7 men) were included in the study. At inclusion, median (quartiles) age and SLE duration were 34 (23.543) and 7 (2-14) years old, respectively. Treatment were hydroxychloroquine (HCQ) $(n=67 ; 64 \%)$, prednisone $(n=66 ; 63 \%)$ with an average $( \pm$ Standard Deviation) dose of $11.3( \pm 16) \mathrm{mg} /$ day and an immunosuppressant $(\mathrm{n}$ $=42 ; 40 \%$ ). According to SFI, 57 SLE patients were experiencing a flare at the time of EBV assessment; flares were classified as severe and mild/ moderate in $38(36 \%)$ and $19(18 \%)$ SLE patients, respectively. According to the SELENA-SLEDAI score, 60 patients $(57 \%)$ were deemed active and $45(43 \%)$ inactive. Main clinical manifestations were arthritis in $32(30 \%)$ patients, constitutional symptoms (fever, weight loss, anorexia or lymphadenopathy) in $31(30 \%)$, cutaneous involvement in $23(22 \%)$, glomerulonephritis in $19(18 \%)$, cytopenia in $14(13 \%)$, neuropsychiatric involvement in 13 $(12 \%)$ and serositis in $10(16 \%)$. Blood EBV DNA was elevated in $54(90 \%)$ of the 60 patients with active lupus versus $6(13 \%)$ of the 45 patients with inactive SLE $\left(\mathrm{p}<10^{-4}\right)$. It was increased in $34(89 \%)$ of the 38 patients with severe flare, in $17(89 \%)$ of the 19 patients with a mild/moderate flare $(p=$ $1)$ and in $8(17 \%)$ of the 48 patients without flare $\left(p<10^{-4}\right.$ vs severe flare and $p<10^{-4}$ vs mild/moderate flare). EBV DNA load correlated with SELENA-SLEDAl score $(r=0.58 ; p<0.0001)$. Elevated blood EBV DNA was not associated with $\mathrm{HCQ}$, prednisone or immunosuppressant intakes. Eighteen patients with active SLE had a second assessment of blood EBV DNA load. For these patients, the median [range] of viral load was significantly higher during periods of active SLE (236 [0-2680] IU/mL) compared with periods with lower SELENA-SLEDAI score $\left(0[0-1537] \mathrm{IU} / \mathrm{mL}, \mathrm{p}<10^{-4}\right.$ in paired analysis)

Conclusion: Blood EBV viral load is dramatically increased in active phase of SLE, independently of the treatment. We were unable to demonstrate whether the replication of EBV was the cause or the consequence or just an epiphenomenon of the disease activity. Further studies are needed to study whether EBV viral load is linked with Interferons secretion or B lymphocyte activation.

Disclosure of Interests: None declared

DOI: 10.1136/annrheumdis-2020-eular.734

\section{FRI0158 \\ CLINICAL AND IMAGING FEATURES OF ARTICULAR MANIFESTATIONS IN PRIMARY SJÖGREN'S SYNDROME: SIMILARITIES AND DIFFERENCES ACCORDING TO THE TIME OF ONSET}

F. Carubbi ${ }^{1}$, A. Alunno ${ }^{2}$, P. Cipriani ${ }^{1}$, E. Bartoloni Bocci ${ }^{2}$, A. Conforti',

I. DI Cola ${ }^{1}$, R. Gerli', R. Giacomelli ${ }^{1} .{ }^{1}$ Rheumatology Unit, L'Aquila, Italy;

${ }^{2}$ Rheumatology Unit, Perugia, Italy

Background: Articular manifestations (AMs) are observed in a large proportion of patients with primary Sjögren's syndrome (pSS) and can occur at the time of pSS diagnosis or during the disease course. Although in the majority of cases AMs are mild and self-limiting, some patients may experience chronic polyarthritis requiring treatment with DMARDs. However to date no specific discriminating biomarkers have been identified. Magnetic resonance imaging (MRI) can help assessing the extent of articular involvement and guide the treatment.

Objectives: To describe clinical and serological features of patients with pSS developing articular involvement along with the MRI findings of affected joints.

Methods: Clinical records were retrospectively evaluated and MRI was performed to evaluate AMs. Disease activity was assessed with the EULAR SS disease activity index (ESSDAI) and its clinical version without the biological domain (ClinESSDAI). Patient-reported symptoms were assessed with the EULAR SS Patient Reported Index (ESSPRI). MRI features were described according to the OMERACT rheumatoid arthritis (RA) MRI scoring system. Values are displayed 
as mean \pm standard error of the mean or number and percentages. Patients were tested for autoantibodies such as anti-cyclic citrullinated peptide, anti-citrullinated a enolase and anti-carbamylated proteins with commercially available ELISA kits.

Results: 45 pSS patients were included. 29 patients (64\%) displayed AMs at pSS onset while $15(36 \%)$ at a later stage (6.7 \pm 1 years after pSS diagnosis). Besides AMs, at the time of pSS diagnosis the two cohorts were comparable with regard to other ESSDAI domains. Interestingly, all patients with anti-SSA and anti-/SSB had AMs at the time of pSS diagnosis $(p=0.05)$ while those developing AMs in the disease course were more likely single positive for anti-SSA $(p=0.04)$. When comparing the clinical and serological features of both groups of patients at the time of overt AMs (Tables 1-2), patients that displayed AMs in the course of the disease have a significantly higher ESSPRI compared to patients that display AMs at pSS onset. With regard to MRI, $80 \%$ of patients with AM displayed signs of synovitis, $59 \%$ bone erosions, $59 \%$ joint space narrowing and $50 \%$ bone marrow oedema. To note, $60 \%$ of patients displaying AMs at pSS onset show bone marrow oedema while this is present in only $27 \%$ of patients developing AMs at a later stage $(p<0.05)$. Anti-cyclic citrullinated peptide, anti-citrullinated a enolase, anti-carbamylated proteins were undetectable in all patients.

Conclusion: Our results confirm the relevance of AMs in pSS, particularly because of the high prevalence of RA-like MRI features. MRI assessment in patients with pSS is advisable to identify more severe AMs in the spectrum of pSS disease and guide the therapeutic approach.

Table 1. Clinical and serological features at the time of overt AMs. Categoric variables

\begin{tabular}{|c|c|c|c|c|c|c|c|}
\hline & \multicolumn{2}{|c|}{$\begin{array}{c}\text { All AMs } \\
(\mathrm{N}=44)\end{array}$} & \multicolumn{2}{|c|}{$\begin{array}{l}\text { AMs at pSS } \\
\text { onset }(n=29)\end{array}$} & \multicolumn{2}{|c|}{$\begin{array}{l}\text { AMs in the } \\
\text { course of } \\
\text { disease } \\
(\mathrm{N}=15)\end{array}$} & \multirow[b]{2}{*}{$\mathrm{p}$ value } \\
\hline & $\mathrm{N}$ & $\%$ & $\mathrm{~N}$ & $\%$ & $\mathrm{~N}$ & $\%$ & \\
\hline \multicolumn{8}{|l|}{ ESSDAI domains } \\
\hline Constitutional & 3 & 7 & 3 & 10 & 0 & 0 & 0.54 \\
\hline Lymphadenopathy & 15 & 34 & 7 & 24 & 8 & 53 & 0.09 \\
\hline Glandular & 9 & 20 & 5 & 17 & 4 & 27 & 0.46 \\
\hline Articular & 44 & 100 & 29 & 100 & 15 & 100 & na \\
\hline Cutaneous & 6 & 14 & 2 & 7 & 4 & 27 & 0.15 \\
\hline Pulmonary & 6 & 14 & 5 & 17 & 1 & 7 & 0.65 \\
\hline Renal & 0 & 0 & 0 & 0 & 0 & 0 & na \\
\hline Muscular & 0 & 0 & 0 & 0 & 0 & 0 & na \\
\hline PNS & 6 & 14 & 4 & 14 & 2 & 13 & 1 \\
\hline CNS & 1 & 2 & 0 & 0 & 1 & 7 & 0.34 \\
\hline Hematological & 10 & 23 & 8 & 28 & 2 & 13 & 0.45 \\
\hline Biological & 5 & 11 & 1 & 3 & 4 & 27 & 0.04 \\
\hline Morning stiffness & 44 & 100 & 29 & 100 & 15 & 100 & na \\
\hline Small joints & 29 & 66 & 17 & 59 & 12 & 80 & 0.31 \\
\hline Large joints & 3 & 7 & 2 & 7 & 1 & 7 & 1 \\
\hline Both & 12 & 27 & 10 & 34 & 2 & 13 & 0.17 \\
\hline
\end{tabular}

Table 2. Clinical and serological features at the time of overt AMs. Continuous variables

\begin{tabular}{lcccc}
\hline & All AMs $(\mathrm{N}=44)$ & $\begin{array}{c}\text { AMs at } \mathrm{pSS} \text { onset } \\
(\mathrm{n}=29)\end{array}$ & $\begin{array}{c}\text { AMs in the course of } \\
\text { disease }(\mathrm{N}=15)\end{array}$ & $\begin{array}{c}\mathrm{p} \\
\text { value }\end{array}$ \\
& MEAN \pm SEM & MEAN \pm SEM & MEAN $\pm S E M$ & \\
\hline Years from pSS & $2.3 \pm 0.6$ & 0 & $6.7 \pm 1$ & na \\
diagnosis & & & & \\
VAS pain & $8.4 \pm 0.3$ & $7.7 \pm 0.4$ & $9.7 \pm 0.15$ & $\mathbf{0 . 0 0 1}$ \\
VAS dryness & $7 \pm 0.4$ & $5.6 \pm 0.5$ & $7.5 \pm 0.6$ & $\mathbf{0 . 0 2}$ \\
VAS fatigue & $6.3 \pm 0.4$ & $6.3 \pm 0.5$ & $8.5 \pm 0.7$ & $\mathbf{0 . 0 2}$ \\
ESSPR & $7.2 \pm 0.3$ & $6.5 \pm 0.4$ & $8.6 \pm 0.4$ & $\mathbf{0 . 0 0 1}$ \\
ESSDAl & $18 \pm 1.6$ & $16.8 \pm 1.8$ & $20.2 \pm 3$ & 0.32 \\
ClinESSDAl & $17.5 \pm 1.6$ & $16.4 \pm 1.8$ & $19.6 \pm 3$ & 0.35 \\
$\mathrm{~N}$ of involved joints & $5.5 \pm 4$ & $6.3 \pm 0.8$ & $4 \pm 0.5$ & 0.06 \\
\end{tabular}

Disclosure of Interests: Francesco Carubbi Speakers bureau: Francesco Carubbi received speaker honoraria from Abbvie and Celgene outside this work., Alessia Alunno: None declared, Paola Cipriani Grant/research support from: Actelion, Pfizer, Speakers bureau: Actelion, Pfizer, Elena Bartoloni Bocci: None declared, Alessandro Conforti: None declared, Ilenia Di Cola: None declared, Roberto Gerli: None declared, Roberto Giacomelli Grant/research support from: Actelion, Pfizer, Speakers bureau: Abbvie, Roche, Actelion, BMS, MSD, Ely Lilly, SOBI, Pfizer

DOI: 10.1136/annrheumdis-2020-eular.6580

\section{FRI0159 EROSIVE ARTHRITIS IN SYSTEMIC LUPUS ERYTHEMATOSUS: APPLICATION OF CLUSTER ANALYSIS}

F. Ceccarelli ${ }^{1}$, F. Natalucci ${ }^{1}$, C. Perricone ${ }^{2}$, E. Cipriano ${ }^{1}$, C. Pirone ${ }^{1}$, G. Olivieri ${ }^{1}$, T. Colasanti ${ }^{1}$, F. R. Spinelli ${ }^{1}$, C. Alessandri ${ }^{1}$, G. Valesini ${ }^{1}$, F. Conti ${ }^{1} .{ }^{1}$ Sapienza Università di Roma, Lupus Clinic, Rheumatology, Rome, Italy; ${ }^{2}$ Università di Perugia, Rheumatology, Perugia, Italy

Background: Systemic Lupus Erythematosus (SLE) related arthritis has been traditionally defined non-erosive and then considered a minor manifestation. Thanks to the application of more sensitive imaging techniques, such as ultrasonography (US), erosive damage has been identified in up to $40 \%$ of SLE patients with joint involvement, suggesting the need for more appropriate treatment (1). Antibodies directed against citrullinated and carbamilated proteins (ACPA and anti-CarP, respectively) have been associated with erosive damage and then proposed as biomarkers for this more aggressive phenotype (2).

Objectives: Here, we evaluated a large SLE cohort with joint involvement by using cluster analysis, in order to identify the disease phenotype associated with erosive arthritis.

Methods: For this analysis, we enrolled consecutive SLE patients (ACR 1997 criteria) with a clinical history of joint involvement (arthritis/arthralgia). Clinical and laboratory data were collected in a standardized computerized electronically filled form, including demographics, past medical history with the date of diagnosis, co-morbidities, previous and concomitant treatments, serological status. The presence of rheumatoid factor (RF), ACPA and anti-CarP was investigated by ELISA test. Erosive damage was assessed by ultrasonography at level of metacarpophalangeal, proximal interphalangeal and metatarsophalangeal joints (MyLab Eight Exp, Esaote, Florence, Italy). Data have been analysed by hierarchic cluster analysis (SPSS program, IBM).

Results: We enrolled 203 patients [M/F 12/191, median age 46.0 years (IQR 18); median disease duration 120.0 months (IQR 108)]. Erosive damage was identified in 53 patients (26.1\%), all of them referring at least one episode of arthritis during disease course. Moving on autoantibodies status, RF was positive in $29.5 \%$, anti-CarP in $28.5 \%$ and ACPA in $11.2 \%$. The univariate analysis demonstrated a significant association between US-detected erosive damage and anti-CarP $(p=0.01)$, ACPA $(p=0.03)$, and renal manifestations $(p=0.03)$. In Figure 1 we reported the dendrogram obtained from cluster analysis, allowing the identification of four cluster. Positivity for ACPA, anti-CarP, erosive damage, Jaccoud's arthropathy and renal manifestations were allocated in the same cluster. Interestingly, RF resulted allocated in a different cluster, including ENA, anti-SSA and anti-SSB antibodies.

Conclusion: The application of cluster analysis allowed the identification of a specific SLE phenotype, characterized by erosive damage, renal manifestations and positivity for anti-CarP and ACPA. We could speculate about the presence of a shared pathogenic mechanism, involving NETosis, contributing to nephritis and erosive arthritis.

References:

[1] Ceccarelli F et al. Semin Arthritis Rheum 2017

[2] Ceccarelli F et al. Arthritis Res Ther 2018

\section{Figure 1: Dendrogram deriving from hierarchical cluster analysis}
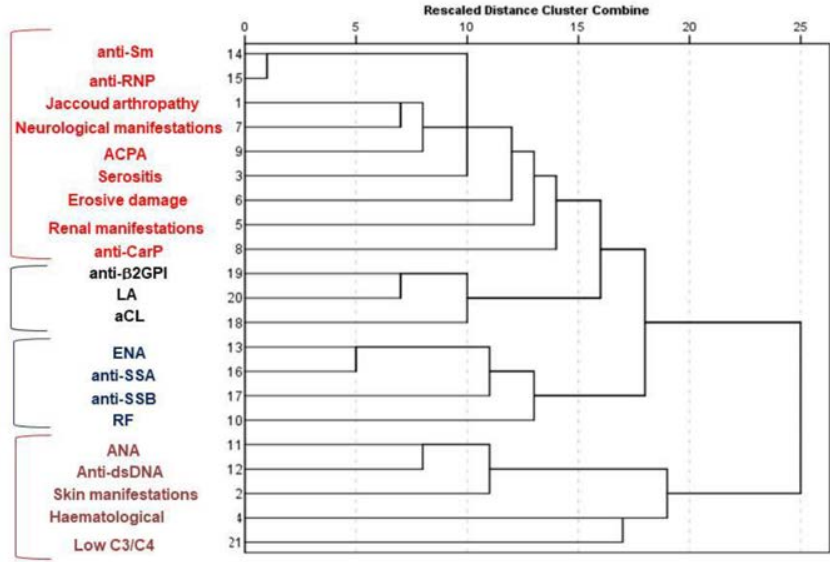

Disclosure of Interests: Fulvia Ceccarelli: None declared, Francesco Natalucci: None declared, Carlo Perricone: None declared, enrica cipriano: None declared, Carmelo Pirone: None declared, Giulio Olivieri: None declared, Tania Colasanti: None declared, Francesca Romana Spinelli Grant/research support from: Pfizer, Consultant of: Novartis, Gilead, Lilly, Sanofi, Celgene, Speakers bureau: Lilly, 